\title{
An Introduction to Superconducting Qubits and Circuit Quantum Electrodynamics
}

\author{
Nicholas Materise
}

\section{Introduction}

Superconducting qubits and circuit quantum electrodynamics have enabled design of solid state sources of quantum information. The performance of these devices has scaled exponentially over the last fifteen years, in terms of their energy relaxation and dephasing times, drawing interest from adjacent communities including the Axion Dark Matter Experiment (ADMX). Recently, superconducting qubits have been targeted for use as single photon detectors in the ADMX high frequency experiment, ADMX-HF [16]. The goal of this article is to give members of the ADMX community an introduction to some of the models used to analyze and design superconducting qubits. This review is not an exhaustive coverage of the field, but it aims to guide the reader to relevant literature and analysis techniques that closely follow experiment.

\section{Superconducting Qubit Circuit Models}

A qubit is a two level system or a system whose controllable quantum dynamics involve its two lowest lying energy levels. Nature provides several forms of qubits or carriers of quantum information including single photons, trapped ions, and atoms in high finesse cavities. Superconducting qubits realize artificial atoms with engineered energy levels using the non-linearity of Josephson junctions and surrounding microwave circuitry [2]. The quantum dynamics of these systems follows that of a damped and driven anharmonic oscillator whose anharmonicity is controlled by choice of circuit parameters, e.g. linear capacitance and inductance of the Josephson junction [15]. For experimental design and control, practitioners draw from the Jaynes-Cummings model and its variants from cavity quantum electrodynamics (QED) [9, 15]. Circuit quantum electrodynamics borrows the application of second quantized Hamiltonians from atomic optics via a standardized procedure for quantizing passive circuit. This section will introduce simple models for Josephson junctions and their role in superconducting qubits. We will then discuss circuit

Nicholas Materise

Lawrence Livermore National Laboratory

7000 East Ave

Livermore, CA 94550

e-mail: materise1ellnl.gov 
quantization methods and Black box quantization techniques used to obtain second quantized Hamiltonians.

\subsection{Non-linearity in Superconducting Qubits}

The operational modes of superconducting qubits vary by their energy spectra, where non-linearity plays a role in realizing accessible and isolated states. If we consider the lowest two levels of the quantum harmonic oscillator to be the ground and excited states of a qubit $(|g\rangle,|e\rangle)$, the energies for the two states are separated by integer multiples of $\hbar \omega$. The classical electric circuit model for an oscillator is the LC circuit, shown in Figure 1. We will refer to this model in Section 2.3 when we derive the second quantized form of the Hamiltonian for an LC circuit. Figure 1 compares the LC oscillator circuit to an anharmonic qubit, the transmon. Notice that the spacing between the excited state $|e\rangle$ and the next highest state $|f\rangle$ is smaller than the spacing between $|g\rangle$ and $|e\rangle$. In more anharmonic oscillators, the spacing is larger, further isolating the qubit states from the other states of the oscillator. The transmon trades off its anharmonicity for reduced sensitivity to charge noise [2].

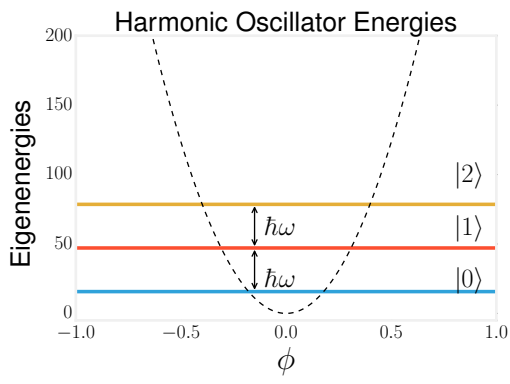

(a)

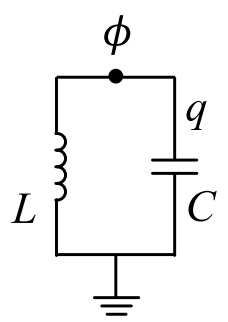

(c)

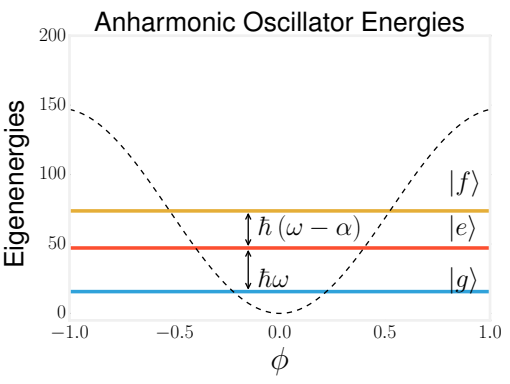

(b)

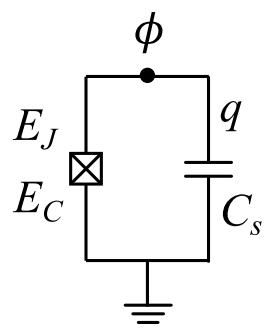

(d)

Fig. 1 Comparison of the quantum harmonic oscillator with anharmonic oscillator. (a) and (b) give the eigenenergies of the two oscillators, where the horizontal lines are the eigenenergies and the dashed lines represent notional potentials. (c) and (d) are the corresponding circuit models for an LC circuit and a transmon qubit [11]. 
An anharmonic oscillator-based superconducting qubit inherits its non-linearity from Josephson junctions, where the non-linearity is tunable through fabrication and microwave circuit design. To develop some intuition for the dynamics of Josephson junctions, we will discuss classical circuit models for the device and their role in superconducting qubits.

\subsection{Classical Circuit Models of Josephson Junctions}

There are several phenomenological models for Josephson junctions that are motivated by the underlying device physics and limits of the electric circuit analogs. We will review the Resistive and Capacitively Shunted Junction (RCSJ) model as outlined in [6].

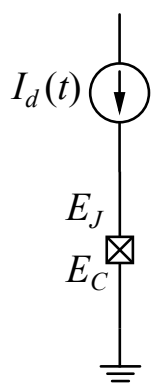

(a)

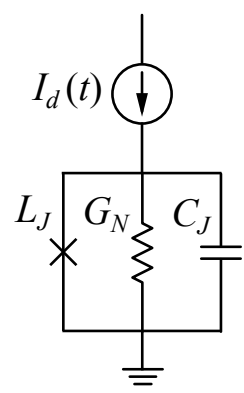

(b)

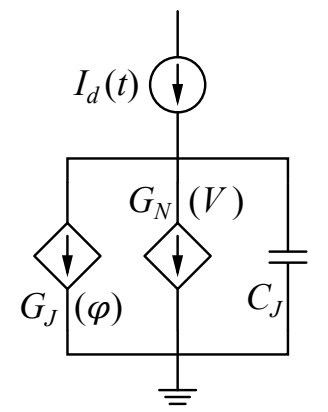

(c)

Fig. 2 (a) Circuit diagram for a current driven Josephson junction, (b) RCSJ circuit model, (c) equivalent circuit with current sources replacing the conductance $G_{N}$ and inductor $L_{J}$

In Figure 2 above, the left most circuit shows a current-driven Josephson with drive current, $I_{d}$. The junction is approximated as the parallel combination of an inductor $L_{J}$, conductance $G_{N}$, and capacitor $C_{J}$. We replace the inductor and conductance with two voltage controlled current sources (VCCS's), $G_{J}(\varphi), G_{N}(V)$, where we use the Gxxx VCCS notation from SPICE [12]. Kirchhoff's current law at the node joining the three circuit elements with the drive current source reads [6]

$$
\begin{array}{r}
I_{d}(t)=I_{c} \sin \varphi+V G_{N}(V)+C_{J} \frac{d V}{d t} \\
G_{N}(V)=\left\{\begin{array}{cc}
0, & |V| \leq 2 \Delta_{0} / e \\
1 / R_{N}, & |V| \geq 2 \Delta_{0} / e
\end{array}\right.
\end{array}
$$

All occurrences of $V$ refer to the voltage across the three elements representing the Josephson junction from the node of their intersection to ground. The super- 
conducting gap energy at zero temperature, $\Delta_{0}$, gives the voltage where the junction transitions from superconducting to a normal metal with a normal resistance $R_{N}$, see Eq. 2 For finite temperatures,(Gross et al. 2016) gives the temperature dependent conductance in the RCSJ based on the the density of states of quasiparticles in the Josephson junction [6].

The VCCS $G_{J}(\varphi)$ varies sinusoidally with the junction phase, $\varphi$, which is a function of the voltage across the junction and given by the Josephson equation

$$
\begin{array}{r}
V=\frac{\Phi_{0}}{2 \pi} \frac{d \varphi}{d t} \\
\Phi_{0}=\frac{h}{2 e} \equiv \text { Magnetic flux quantum }
\end{array}
$$

If we substitute Eq. 3 into Eq. 1, we arrive at a second order linear differential equation in the phase variable, $\varphi$

$$
I_{d}(t)=\frac{\Phi_{0}}{2 \pi} C_{J} \frac{d^{2} \varphi}{d t^{2}}+\frac{\Phi_{0}}{2 \pi} \frac{d \varphi}{d t} G_{N}\left(\frac{\Phi_{0}}{2 \pi} \frac{d \varphi}{d t}\right)+I_{c} \sin \varphi
$$

This equation is analogous to a driven pendulum, where the capacitance and conductance are proportional to the mass and damping parameter for the pendulum, respectively [15]. For practical, classical simulations of Josephson junctions, the two VCCS model shunted by the junction capacitance is sufficient to produce hysteresis in the current-voltage (IV) characteristic curve. Numerical simulation of the circuit in Figure 2] is well suited for SPICE [12] circuit solvers or coupled to geometries in multiphysics codes such as COMSOL [5].

The RCSJ model is an intuitive model for the behavior of a Josephson junction with an applied dc or ac drive current, though it is not as suitable for superconducting qubit design and simulation. Circuit Quantum Electrodynamics provides a framework analyzing such systems with the language of atomic optics or cavity quantum electrodynamics. We will examine the key features of circuit QED and its utility in the design and simulation of superconducting qubits.

\subsection{Circuit Quantum Electrodynamics}

Circuit quantum electrodynamics (QED) combines microwave engineering, circuit analysis, and quantum optics. Fabry-Perot cavities from optics are replaced by resonant microwave cavities or lumped element microwave resonators in circuit QED. The procedure for obtaining the quantized Hamiltonian and subsequent dynamics of the system follows first from a classical treatment, then quantization of the classical variables as operators and relating those operators to bosonic single-mode raising and lowering operators $\left\{\hat{a}_{i}^{(\dagger)}\right\}$. 


\subsubsection{Quantizing the LC Oscillator}

We return to the LC oscillator circuit in Figure 1 and write the Lagrangian for the circuit in terms of the flux variable $\phi$ which is treated as the generalized coordinate for the system [4].

$$
\mathcal{L}(\phi, \dot{\phi})=\frac{1}{2} C \dot{\phi}^{2}-\frac{1}{2 L} \phi^{2}
$$

We treat the charge $q$ on the capacitor as the conjugate momentum and perform a Legendre transformation to obtain the Hamiltonian as a function of both $q$ and $\phi$.

$$
\begin{array}{r}
q=\frac{\partial \mathcal{L}}{\partial \dot{\phi}}=C \dot{\phi} \Longrightarrow \dot{\phi}^{2}=q^{2} / C^{2} \\
\mathcal{H}(q, \phi)=\dot{\phi} q-\mathcal{L}=\frac{1}{2} C \dot{\phi}^{2}+\frac{1}{2 L} \phi^{2} \\
\mathcal{H}=\frac{1}{2 C} q^{2}+\frac{1}{2 L} \phi^{2}
\end{array}
$$

Following the example in Chapter 3 of [15], the charge and flux variables are quantized by converting them to operators with the commutation relation $[\hat{\phi}, \hat{q}]=$ $i \hbar$. If we take the resonance frequency of the LC circuit to be $\omega=(L C)^{-1 / 2}$ and replace $1 / L$ in the potential term of the Hamiltonian, we arrive at the familiar form for a harmonic oscillator with mass $C$.

$$
\mathcal{H} \rightarrow \hat{H}=\frac{\hat{q}^{2}}{2 C}+\frac{1}{2} C \omega^{2} \hat{\phi}^{2}
$$

We define raising and lowering operators for this quantum harmonic oscillator in analogy to those used in the one-dimensional model and write the second quantized form of the Hamiltonian.

$$
\begin{array}{r}
\hat{q}=-i \sqrt{\frac{\hbar \omega C}{2}}\left(\hat{a}-\hat{a}^{\dagger}\right), \quad \hat{\phi}=\sqrt{\frac{\hbar}{2 \omega C}}\left(\hat{a}+\hat{a}^{\dagger}\right) \\
\hat{H}=\hbar \omega\left(\hat{a}^{\dagger} \hat{a}+1 / 2\right)
\end{array}
$$

\subsubsection{Black Box Circuit Quantization}

In the previous section, we covered a procedure for quantizing an LC oscillator circuit which leads to an approximate generalization for any device given its frequency dependent impedance function. This approach connects full wave electromagnetic simulations of microwave circuits to their quantum mechanical analogs in circuit QED. Given a single port $S$-parameter as a function of frequency, one can obtain the impedance at the port by the transformation 


$$
\begin{array}{r}
Z=(\mathbb{1}+S)(\mathbb{1}-S)^{-1} \\
\mathbb{1} \equiv \text { identity matrix with same dimensions as } S
\end{array}
$$

Following the Black box quantization methods outlined in [13, 17], the impedance function, $Z(\omega)$ can be expressed as a pole-residue expansion in the complex frequency $s=j \omega$, where $j=-\sqrt{-1}$, following the electrical engineering convention.

$$
\begin{array}{r}
Z(s)=\sum_{k=1}^{M} \frac{r_{k}}{s-s_{k}}+d+e s \\
\left\{r_{k}=a_{k}+j b_{k}\right\} \equiv \text { residues, } \quad\left\{s_{k}=\xi_{k}+j \omega_{k}\right\} \equiv \text { poles }
\end{array}
$$

The above rational function can be obtained by a least squares fit of the original impedance using the Vector Fit software outlined in [7] and available at [18]. If we take the case where $d=0$ and the pole at $s \rightarrow \infty$ vanishes or $e=0$ and perform the following partial fraction expansion and approximation for the $k$-th term in the series and we find the $k$-th term is the impedance for a parallel RLC oscillator circuit.

$$
\begin{array}{r}
Z_{k}(s)=\frac{r_{k}}{s-s_{k}}=\frac{r_{k}}{s-s_{k}}+\frac{r_{k}^{*}}{s-s_{k}^{*}} \simeq \frac{2 a_{k} s}{s^{2}-2 \xi_{k} s+\omega_{k}^{2}} \\
\Longrightarrow Z_{k}(s)=\frac{\frac{\omega_{k} r_{k}}{Q_{k}} s}{s^{2}+\frac{\omega_{k}}{Q_{k}} s+\omega_{k}^{2}} \\
\omega_{k}=\left(L_{k} C_{k}\right)^{-1 / 2}, \quad Q_{k}=\omega_{k} R_{k} C_{k}=-\omega_{k} / 2 \xi_{k}, \quad R_{k}=-a_{k} / \xi_{k}
\end{array}
$$

The total impedance, $Z(s)$ is a series combination of RLC oscillators and if we take the dissipationless limit by ignoring the resistances, we can treat $Z(s)$ as a series combination of LC circuits and apply the same analysis from Section 2.3.1 to each subcircuit. If we shunt the resulting circuit, with a single Josephson junction, we can obtain a simple model for the Hamiltonian of a qubit coupled to a superconducting resonator with $M$-modes. For a full derivation of the non-linear components of the Hamiltonian $\hat{H}_{\mathrm{nl}}$, see [13]; we reproduce the salient features here.

$$
\begin{array}{r}
\hat{H}=\hat{H}_{0}+\hat{H}_{\mathrm{nl}} \\
\hat{H}_{0}=\sum_{i} \hbar \omega_{i} \hat{a}_{i}^{\dagger} \hat{a}_{i}, \quad \hat{H}_{\mathrm{nl}}=E_{J}\left(1-\cos \hat{\varphi}-\frac{\hat{\varphi}^{2}}{2}\right) \\
\hat{H}_{\mathrm{nl}} \approx-\frac{1}{2} \sum_{i} \alpha_{i} \hat{a}_{i}^{\dagger 2} \hat{a}_{i}^{2}-\sum_{i \neq j} \chi_{i j} \hat{a}_{i}^{\dagger} \hat{a}_{i} \hat{a}_{j}^{\dagger} \hat{a}_{j} \\
\hat{\varphi}=\frac{2 \pi}{\Phi_{0}} \sum_{i} \hat{\phi}_{i}=\frac{2 \pi}{\Phi_{0}} \sum_{i} \sqrt{\frac{\hbar}{2 \omega_{i} C_{i}}}\left(\hat{a}_{i}+\hat{a}_{i}^{\dagger}\right)
\end{array}
$$


The Hamiltonian above is referred to as the dispersive Hamiltonian for a weakly anharmonic qubit coupled to a series of harmonic modes. In the non-linear term, $\hat{H}_{\mathrm{nl}}$, the first contribution describes the anharmonicities of those modes and the qubit mode or self-Kerr terms and the second term gives the cross-Kerr terms [13]. Both $\left\{\alpha_{i}\right\}$ and $\left\{\chi_{i j}\right\}$ are experimentally observable, tying this model for qubit-circuit interactions to physical devices.

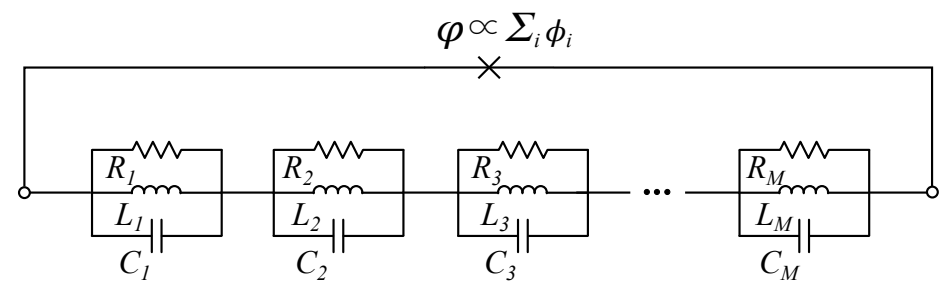

Fig. 3 Series combination of RLC circuits shunted by a single Josephson junction representing the black box circuit from Eq. 12 and similar in design to the circuit in [3]

\section{Summary}

The models used to describe the operation of superconducting qubits follow intuitive modifications to the familiar damped and driven oscillator systems from classical and quantum mechanics. These models arise from careful application of circuit QED to incorporate the quantum effects of macroscopic structures in microwave circuits. Although the dispersive Hamiltonian describes many superconducting qubit systems in quantum information experiments, this article did not apply the model to the problem single photon counting. For more resources related to circuit QED and single photon counting, please refer to [4, 15, 1, 10, 14, 8].

\section{Acknowledgements}

This work was performed under the auspices of the U.S. Department of Energy by Lawrence Livermore National Laboratory under Contract DE-AC52-07NA27344 and funded by the Laboratory Directed Research and Development programs at LLNL project numbers 15-ERD-051, 16-SI-004. 


\section{References}

1. L. Bishop. Circuit Quantum Electrodynamics. PhD thesis, Yale University, 2010.

2. A. Blais, R.-S. Huang, A. Wallraff, S. M. Girvin, and R. J. Schoelkopf. Cavity quantum electrodynamics for superconducting electrical circuits: An architecture for quantum computation. Phys. Rev. A, 69:062320, Jun 2004.

3. J. Bourassa, F. Beaudoin, J. M. Gambetta, and A. Blais. Josephson-junction-embedded transmission-line resonators: From Kerr medium to in-line transmon. Phys. Rev. A, 86:013814, Jul 2012.

4. A. A. Clerk, M. H. Devoret, S. M. Girvin, F. Marquardt, and R. J. Schoelkopf. Introduction to quantum noise, measurement, and amplification. Rev. Mod. Phys., 82:1155-1208, Apr 2010.

5. COMSOL Multiphysics. WWW. comsol.com

6. R. Gross, A. Marx, and F. Deppe. Applied Superconductivity: Josephson Effect and Superconducting Electronics. Walter de Gruyter, 2016.

7. B. Gustavsen and A. Semlyen. Rational approximation of frequency domain responses by vector fitting. IEEE Transactions on Power Delivery, 14(3):1052-1061, Jul 1999.

8. E. Holland. Cavity State Reservoir Engineering in Circuit Quantum Electrodynamics. PhD thesis, Yale University, 2015.

9. E. T. Jaynes and F. W. Cummings. Comparison of quantum and semiclassical radiation theories with application to the beam maser. Proceedings of the IEEE, 51(1):89-109, Jan 1963.

10. B. Johnson. Controlling Photons in Superconducting Electrical Circuits. PhD thesis, Yale University, 2011.

11. J. Koch, T. M. Yu, J. Gambetta, A. A. Houck, D. I. Schuster, J. Majer, A. Blais, M. H. Devoret, S. M. Girvin, and R. J. Schoelkopf. Charge-insensitive qubit design derived from the Cooper pair box. Phys. Rev. A, 76:042319, Oct 2007.

12. L. W. Nagel and D. Pederson. SPICE (Simulation Program with Integrated Circuit Emphasis). Technical Report UCB/ERL M382, EECS Department, University of California, Berkeley, Apr 1973.

13. S. E. Nigg, H. Paik, B. Vlastakis, G. Kirchmair, S. Shankar, L. Frunzio, M. H. Devoret, R. J. Schoelkopf, and S. M. Girvin. Black-Box Superconducting Circuit Quantization. Phys. Rev. Lett., 108:240502, Jun 2012.

14. M. Reed. Entanglement and Quantum Error Correction with Superconducting Qubits. PhD thesis, Yale University, 2013.

15. D. Schuster. Circuit Quantum Electrodynamics. PhD thesis, Yale University, 2007.

16. T. M. Shokair, J. Root, K. A. Van Bibber, B. Brubaker, Y. V. Gurevich, S. B. Cahn, S. K. Lamoreaux, M. A. Anil, K. W. Lehnert, B. K. Mitchell, A. Reed, and G. Carosi. Future directions in the microwave cavity search for dark matter axions. International Journal of Modern Physics A, 29(19):1443004, 2014.

17. F. Solgun, D. W. Abraham, and D. P. DiVincenzo. Blackbox quantization of superconducting circuits using exact impedance synthesis. Phys. Rev. B, 90:134504, Oct 2014.

18. The Vector Fitting Web Site-SINTEF.https://www.sintef .no/projectweb/vectfit/ 